\title{
Oncocytic Adrenocortical Carcinoma: A Rare Case Report
}

\author{
(1) Oktay Üçer MD¹, (1) Oğuzcan Erbatu MD', (1) Ayça Tan MD², (1) Talha Müezzinoğlu MD¹ \\ 'Celal Bayar University Faculty of Medicine, Department of Urology, Manisa, Turkey \\ ${ }^{2}$ Celal Bayar University Faculty of Medicine, Department of Pathology, Manisa, Turkey
}

\begin{abstract}
Oncocytic neoplasms of the adrenal cortex are uncommon and generally benign and non-functioning. About $20 \%$ of adrenocortical oncocytic neoplasms show malignant components. A 39-year-old woman with an adrenocortical oncocytic carcinoma is reported in this article. The patient presented with mild right-sided pain. Computed tomography (CT) showed a $3 \mathrm{~cm} \times 2 \mathrm{~cm}$ tumor in the right adrenal gland. The mass was atypical for adrenal adenoma and follow-up was recommended. Follow-up CT after 1 year showed tumor growth $(5 \mathrm{~cm})$ and the patient underwent laparoscopic surgery for pathologic verification. She was diagnosed as oncocytic adrenocortical carcinoma based on the pathologic diagnostic criteria. Due to their rarity, especially in cases of malignancy, there are no clear treatment and follow-up protocols. Curative surgical treatment should aim for complete excision of the tumor. As the literature about these tumors develops, more information about the nature of the disease will come to light.
\end{abstract}

Keywords: Oncocytic neoplasm, adrenal cortex, oncocytic adrenocortical carcinoma, laparoscopic adrenalectomy

\section{Introduction}

Oncocytic neoplasms can be seen in various organs, but they are usually detected in the thyroid, salivary glands, and kidneys. They contain oncocytic tumor cells that are recognized by their broad, eosinophilic and granular cytoplasm due to anomalous mitochondrial collection (1). Oncocytic neoplasms of the adrenal gland are uncommon and are generally benign and nonfunctioning. These tumors are more common in women and on the left adrenal gland. To date, 147 cases have been reported (2). Recent reports indicate that about $20 \%$ of the adrenocortical oncocytic neoplasms show malignant components and 10-20\% produce hormones which may cause symptoms of Cushing's syndrome or virilism (3).

The most recent classification according to histopathological features is the Weiss criteria modified by Aubert. The Weiss criteria include high nuclear grade, more than 5 mitoses per 50 high-power fields, atypical mitosis, $<25 \%$ clear cells, diffuse architecture, indeterminate, sinusoidal or capsular invasion. A malignant diagnosis is made in the presence of 3 or more of these criteria (4).

Bisceglia et al. (5) used a new method to classify oncocytic adrenocortical neoplasms. In this algorithm, more than 5 mitoses per 50 high-power fields, atypical mitotic figures, and venous invasion were identified as major criteria. The presence of necrosis, capsular invasion, and sinusoidal invasion were defined as minor criteria, with a diameter exceeding $10 \mathrm{~cm}$ and/or weight exceeding 200 grams. Tumors with one major criterion are considered malignant. Tumors with 1 to 4 minor criteria are considered to have indeterminate malignancy potential. Tumors that do not meet any of the criteria are defined as benign (5).

\section{Case Presentation}

Herein we present a 39-year-old woman with an adrenocortical oncocytic carcinoma. The patient underwent surgery for a 5 $\mathrm{cm}$ right-sided adrenal tumor. The patient provided informed consent for this article. 
In August 2016, a 39-year-old woman with complaints of mild right-sided pain was admitted to hospital. Computed tomography (CT) showed a $3 \mathrm{~cm} \times 2 \mathrm{~cm}$ tumor in the right adrenal gland. The mass had atypical density for a usual adrenal adenoma and follow-up was recommended.

Physical examination revealed no specific findings of any urological or endocrine system disease. In laboratory tests, the patient's blood count values, serum electrolytes, and glucose levels were within normal ranges. Hormone tests were performed to determine if the tumor was active. No anomalies were detected in morning plasma cortisol and adrenocorticotropic hormone levels, thyroid hormones, or creatinine levels. There were no signs of hepatic invasion in radiological imaging or in serum levels such as aspartate amino transferase, alanine amino transferase, international normalized ratio, and other liver function tests. Her blood pressure was also normal. She had been operated for a benign breast cyst approximately ten years earlier.

She was under active surveillance for a year. In July 2017, magnetic resonance imaging (MRI) revealed a $4 \times 4 \times 3 \mathrm{~cm}$ right adrenal mass with an apparent diffusion coefficient value of $1.01 \times 10^{-3} \mathrm{~mm}^{2} / \mathrm{s}$ and signal loss of $10 \%$. The mass showed heterogeneous contrast enhancement on MRI and was atypical for adrenal adenoma. Hormone tests to determine tumor activity were repeated and again showed no anomalies.

Another CT scan done before adrenalectomy (Figure 1) showed the same dimensions as the last MRI. The mass had no invasion to any vasculature or to the liver. Its borders were well defined and washout was measured as $61.5 \%$, which previous reports have suggested may indicate a lipid-poor adenoma. There was no organ metastasis according to the last screenings. She underwent laparoscopic adrenalectomy.

The patient was diagnosed with oncocytic adrenocortical carcinoma according to the method used by Bisceglia et al. (5). The mass was $5 \mathrm{~cm}$ at its maximum diameter and weighed 46 grams with a slim capsule. The tumor consisted of polygonal oncocytes with granular, eosinophilic cytoplasm (Figure 2). The mitotic rate was 5 per 50 high-power fields. The tumor had atypical mitoses with no necrosis. No capsular, vascular, or sinusoidal invasion was detected.

The patient remains under postoperative follow-up. Three months after the operation, she underwent blood tests and cross-sectional imaging, as well as positron-emitting tomography (PET) scan which showed no evidence of residual mass or distant metastases. The patient continues to be closely

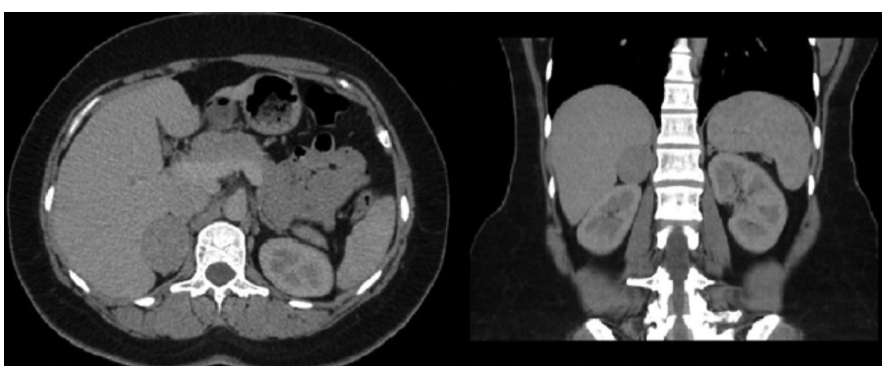

Figure 1. Contrast computed tomography image of the adrenocortical oncocytic carcinoma

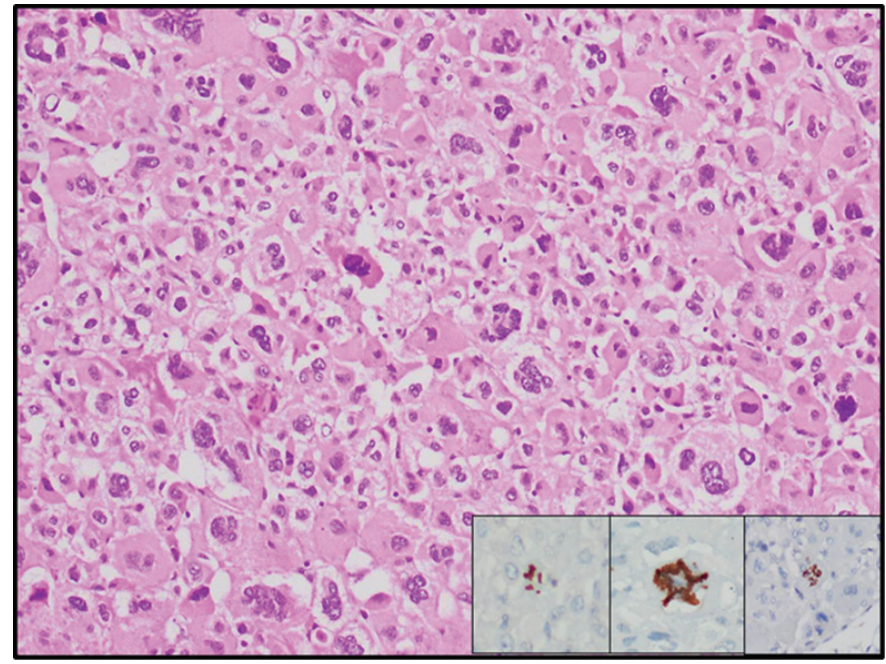

Figure 2. The tumor is composed of oncocytic cells with eosinophilic cytoplasm and nuclear pleomorphism; inset: distinctive atypical mitotic figures

monitored and performance assessment after discharge is highly encouraging.

\section{Discussion}

Oncocytic neoplasms of the adrenal cortex are uncommon, and are generally benign and non-functioning. According to latest reports, about $20 \%$ of adrenocortical oncocytic neoplasms show malignancy and $10-20 \%$ produce hormones (3).

In our case, the patient was diagnosed with oncocytic adrenocortical carcinoma according to the method described by Bisceglia et al. (5). There was a positive finding of atypical mitotic figures, which is a major component. There was no finding compatible with any other criteria, major or minor.

With current technology, these types of tumors cannot be definitively diagnosed by CT or MRI without obtaining the necessary histopathologic samples. If possible, laparoscopic adrenalectomy is still the only option. Since there are limited examples in the literature and there is a lack of experience, it is still not possible to make definite interpretations about pre- and postoperative approaches.

Regarding diagnosis and follow-up, apart from conventional cross-sectional imaging methods, PET appears to have an increasing prevalence. Although shown in a limited number of studies, it has been shown that 18-fluorine-fluorodeoxyglucose (18F-FDG) PET is effective for benign/malignant differentiation in radiographically and pathologically diagnosed oncocytic adrenal masses. It is also a valuable test in terms of distant metastasis and recurrence. It should be remembered that these tumors can show increased 18F-FDG uptake, which would bring a new perspective to the subject (6).

A follow-up period of at least 5 years after surgery is recommended. However, oncocytic adrenocortical carcinoma can invade nearby tissues or metastasize to distant organs. The 5-year survival rate is $50-60 \%$ after standard surgical excision, but if the patient is not a surgical candidate, radiotherapy or chemotherapy are palliative or curative options (2). 
Oncocytic neoplasms of the adrenal cortex are uncommon and generally non-functioning and benign. However, a small proportion of them exhibit malignant features. Due to their rarity, especially in malignant cases, there is no definitive treatment method. Curative surgical treatment should aim for complete excision of the tumor. In selected cases, radiotherapy or chemotherapy with cytotoxic drugs could be a palliative or curative option.

As the literature concerning these rare tumors develops, the complexity of diagnosis, treatment, and follow-up will be reduced and more accurate information about the nature of the disease will come to light.

\section{Ethics}

Informed Consent: Informed written consent was obtained from the patient for the writing of the case presentation containing pathological diagnosis and follow-up information.

Peer-review: Externally peer-reviewed.

\section{Authorship Contributions}

Surgical and Medical Practices: O.Ü., T.M., Concept: O.Ü., Design: O.E., O.Ü., Data Collection or Processing: T.M., O.Ü., Analysis or Interpretation: T.M., O.Ü., Literature Search: T.M., A.T., Writing: O.E.
Conflict of Interest: No conflict of interest was declared by the authors.

Financial Disclosure: The authors declared that this study received no financial support.

\section{References}

1. Alamoudi O, Alsulaiman W, Aldhaam N, Moazin M. Incidental Finding of Adrenal Oncocytoma After Right Robotic Adrenalectomy: Case Report and Literature Review. Urol Case Rep 2017;12:14-16.

2. Mearini L, Del Sordo R, Costantini E, et al. Adrenal Oncocytic Neoplasm: A Systematic Review. Urol Int 2013;91:125-133.

3. Kabayegit OY, Soysal D, Oruk G, et al. Adrenocortical oncocytic neoplasm presenting with Cushing's syndrome a case report. J Med Case Rep 2008;2:228.

4. Weiss LM. Comparative histological study of 43 metastasizing and nonmetastasizing adrenocortical tumors. Am J Surg Pathol 1984;8:163-169.

5. Bisceglia M, Ludovico O, Di Mattia A, et al. Adrenocortical oncocytic tumors: report of 10 cases and review of the literature. Int J Surg Pathol 2004;12:231-243.

6. Kim DJ, Chung JJ, Ryu YH, et al. Adrenocortical oncocytoma displaying intense activity on 18F-FDG-PET: a case report and a literature review. Ann Nucl Med 2008;22:821-824. 